\title{
An Empirical Case of Chinese Medicine in the Treatment of Idiopathic Edema
}

\author{
Xiaojuan Wang1, Xiaohui Li ${ }^{2 *}$ \\ 1Shaanxi University of Chinese Medicine, Xianyang 712000, Shaanxi Province, China \\ ${ }^{2}$ The Affiliated Hospital of Shaanxi University of Chinese Medicine, Xianyang 712000, Shaanxi Province, China \\ *Corresponding author: Xiaohui Li, Jyhtt20050418@126.com
}

\begin{abstract}
Idiopathic edema is a common, challenging, and complicated clinical condition with no recognized cause that is easy to prolong and repeat. Professor Li has a lot of expertise treating this condition. This condition is associated with a yang shortage in the spleen and kidneys, as well as a mutual accumulation of water and blood stasis. Zhenwu Decoction and Guizhi Fuling tablet are added to the prescription. Ms. Li's clinical experience in the treatment of idiopathic edema is being shared with the fellow.
\end{abstract}

Keywords: Idiopathic edema; Traditional Chinese medicine; Clinical experience

Publication date: November 2021; Online publication: November 30, 2021

\section{Introduction}

One of the types of edema is idiopathic edema, which is a clinical condition. The occurrence of this condition is mostly caused by a disturbance in the patients' water and salt metabolism as a result of a variety of circumstances ${ }^{[1]}$. Endocrine disorders, mood swings, age, and the venous reflux barrier are all common causes ${ }^{[2]}$. The pathophysiology of this condition, which is more common in women, particularly perimenopausal women, remains unknown. Edema of the eyes, face, and lower limbs were the most common clinical symptoms. In traditional Chinese medicine, idiopathic edema is classified as a "edoema illness." The pathogenesis includes external pathogens, poor diet, extreme weariness, and other factors that lead to lung, spleen, kidney, and triple energizer malfunction. For more than 20 years, Ms. Li has worked in the field of nephropathy clinical research. He has devoted himself to the study of classical prescriptions for the treatment of chronic diseases and accumulated rich clinical experience in the treatment of various chronic diseases. A case of treating idiopathic edema based on syndrome differentiation of traditional Chinese medicine has achieved remarkable therapeutic effect. The records are as follows:

The patient, female, 58 years old, was initially diagnosed on April 14, 2020. Principal complaint: Intermittent edema for 1 year and recur for another 5 days. Current medical history: Eyelid edema gradually affected the face and both lower limbs for 1 year without inducement. 5 days ago, edema of face, eyelids and lower limbs recurred, so she came to see a doctor. Symptoms could be seen as fellow: Fatigue, slight edema of face, eyelids and lower limbs, light complexion, backache and pain, slightly dry mouth, dizziness and headache, poor diet, poor night rest, chilly, moderate urine output, no frequent urination, urgent urination, odynuria and gross hematuria, normal stool, light and dark tongue, fat tongue, white, thick and greasy fur, heavy pulse, thin and smooth string. Laboratory inspection was: blood routine, urine routine, liver function, kidney function, electrolyte and nail function, and all were normal. Western medcine diagnosis was: idiopathic edema. TCM diagnosis was: edema, syndrome differentiation was spleen and kidney yang deficiency, water and blood stasis syndrome. It was advisable to strengthen the spleen and 
kidney, promote blood circulation and water. The prescription was Zhenwu decoction combined with Guizhi Fuling pill. The prescription was: Poria cocos 15g, Radix Paeoniae Alba 10g, Radix Paeoniae Rubra $10 \mathrm{~g}$, ginger peel $12 \mathrm{~g}$, black sliced aconite $10 \mathrm{~g}$, fried Rhizoma Atractylodis Macrocephalae 12g, Ramulus Cinnamomiand 15g, peach seed 12g, paeonia suffruticosa 9g, Radix Angelicae Sinensis 12g, Radix Bupleuri 12g, Radix Astragali seu Hedysari 12g. 15 doses, 1 dose each day, the perscription can be fried in water for 2 times, taken $300 \mathrm{ml}$ extract, and taken with warm water in the morning and evening. On May 3 , 2020, the second diagnosis showed that the patient had less edema of eyelids, face and lower limbs, no fatigue, no backache and pain, improved appetite, well night rest, no dizziness, headache, light red tongue, thin white and greasy fur and thin pulse. She was instructed to continue taking traditional Chinese medicine and rechecked regularly for 1 year. There was no recurrence of edema.

The treatment of edema focused on facilitating lung, strengthening the spleen and benefiting the kidney, especially the kidney. According to the clinical symptoms, it could be classified into the category of "edema disease". Edema disease was called "water" in "Neijing," and could be divided into "Feng Shui", "Shi Shui" and "Yong Shui" according to different symptoms. Many factors such as exogenous wind, cold, dampness, internal injury, diet, seven emotions, and long illness, fatigue, etc. caused pulmonary dysfunction, spleen failure, and kidney failure and leaded to water retention in the body, overflow skin a series of symptoms. Edema disease is often mixed with deficiency and excess and occurs repeatedly. Deficiency syndrome refers to Qi deficiency of lung, spleen and kidney. Repletion syndrome refers to blood stasis, Qi stagnation, water dampness, turbid poison, etc. In terms of clinical symptoms, edema was easy to occur due to internal causes such as insufficient endowment, improper diet, long illness and fatigue, as well as the invasion of external pathogens such as wind evil, water dampness and sore poison. In view of its etiology and pathogenesis, Su Wen · Shui re acupoint theory pointed out that: "Therefore, its origin lied in the kidney and its end lies in the lung. [3] "Plain Questions, Treatise on Points for Water and Heat" also pointed out: "All dampness and swelling belonged to the spleen." For the treatment of edema, Su Wen · Tang Ye Lao Li Lun put forward that: The treatment principle of "peaceful treatment depended on balance, got rid of the bad stuff that has been accumulating in the body.... Open the Huimen and clean the Jingfu" [4].

The synopsis of the "Synopsis of Golden Chamber" is divided into five types: wind edema, skinedema, typical anasarca, stony edema and yellow sweat. According to the pathogenesis and syndrome of the five internal organs, the book divides edema into heart water, liver water, lung water, spleen water and kidney water. In terms of treatment, the indications of sweating and diuresis are put forward that: "For those who have edema, swelling below the waist should relieve urine; swelling above the waist should heal when sweating." "Beiji Qianjin Yaofang" pointed out for the first time that salt must be avoided for edema disease. Yonghe Yan also advocated the method of warming the spleen and kidney in the treatment of edema. The theory of "Renzhai Zhizhi Fang · Xu Zhong Fang" established the method of promoting blood circulation and diuresis to treat blood stasis and edema. If the patient's condition is repeated, the healthy Qi is damaged, the Qi deficiency is unable to promote the operation of water and liquid, and the dampness evil is blocked, edema, fatigue and fatigue can be seen; If the body fluid is not superior to the mouth, the mouth is slightly dry. Radix Astragali seu Hedysari, Poria cocos and ginger peel are given to strengthen the spleen and replenish Qi, support healthy Qi, promote Qi and water, promote the healthy movement of the spleen, then the water dampness will not overflow, and the edema will disappear when Qi moves. If the kidney essence is lost due to chronic disease and physical deficiency, and there is no way to nourish the muscles and veins of the waist, the waist will be sour and painful; If Qi deficiency lasts for a long time and Yang Qi is damaged, you will be afraid of cold and look plaint. Give black sliced aconite, Ramulus Cinnamomiand and Radix Angelicae Sinensis to warm the kidney, benefit essence, nourish blood and dredge the pulse; Menstruation has disappeared, kidney Qi is gradually declining, life gate fire is declining, Chongren is in disorder, the spleen and stomach is damage, appetite is poor and night rest is poor; Deficiency of spleen and kidney and 
loss of brain support will cause dizziness and headache. Rhizoma Atractylodis Macrocephalae can nourish the liver and soften the liver, prevent too much water from damaging Yin, bupleurum can soothe the liver and regulate Qi, Rhizoma Atractylodis Macrocephalae can strengthen the spleen, replenish Qi, benefit water and reduce swelling; A long illness came into stasis, as the synopsis of the Golden Chamber says: "If blood is not inducing, it is water "5]." Give paeonia suffruticosa, peach seed and Radix Angelicae Sinensis to activate blood circulation and remove blood stasis, nourish blood and dredge collaterals. The tongue is light and dark, the tongue body is fat, the moss is white, thick and greasy, the pulse is heavy, and the string is fine and slippery, which is the features of Yang deficiency of spleen and kidney, water and blood stasis. All drugs play the functions of strengthening the spleen and warming the kidney, promoting water and detumescence, promoting blood circulation and removing blood stasis. The prognosis of this patient was better after TCM syndrome differentiation.

\section{Disclosure statement}

The authors declare no conflict of interest.

\section{References}

[1] Wu A, Zhao J, 2019, Treatment of Idiopathic Edema from the Five Internal Organs. World Latest Medicine Information, 19(96): $245+248$.

[2] Liu T, 2017, Dr. Li Shiping's Experience in the Treatment of Idiopathic Edema. Shaanxi Journal of Traditional Chinese Medicine, 38(09): 1277-1278.

[3] Yan Y, Zhao F, Xiao Y, et al., 2016, Discussion on Chronic Glomerulonephritis from "the Root is in the Kidney and the End is in the Lung". Chinese Medicine Modern Distance Education of China, 14(08): 59-61.

[4] Yan P, Zhuang X, Ai S, et al., 2021, A Brief Discussion on the Treatment of Primary Nephrotic Syndrome in Children from "Opening the Guimen, Ceaning the Jingfu and Removing the Bad Stuff that Has Been Accumulating in the Body. Lishizhen Medicine and Materia Medica Research, 32(04): 931-932.

[5] Ma Y, Zhang B, Li Q, et al., 2018, Professor Jiang Bohua's Experience in Treating Women's Idiopathic Edema from the Perspective of "Blood which is not Inducing is Water". Heilongjiang Journal of Traditional Chinese Medicine, 47(06): 62-63. 